\title{
Calculation of Temperature Distribution in Eccentric Multi Core Diode Pumped Fiber Lasers by Green Function Method
}

\author{
A.R. GharaAti ${ }^{a}, *$ P. Elahi ${ }^{b}$, M. JAFAri $^{a}$ \\ ${ }^{a}$ Department of Physics, College of Science, Payme Noor University (PNU), Shiraz, Iran. \\ ${ }^{b}$ Department of Physics, College of Science, Shiraz University of Technology, Shiraz, Iran
}

In this paper, the temperature distribution in fiber lasers pumped by multi diode bars located in out-of-fiber axis has been investigated semi-analytically. For this reason, we consider heat deposition in fiber lasers due to pump and the nonhomogeneous heat conduction equation has been solved by using Green function method in a cylindrical coordinate system. Our model has been used for single, double and multi pumped configuration and, for a typical fiber lasers, the results have been plotted and discussed.

PACS numbers: 42.55.Wd, 42.60.Da

\section{Introduction}

Fiber lasers have been the subject of considerable attention recently [1-3], because they operate in low threshold, have high efficiency, high beam quality, and can produce a single mode beam [4-8]. In power-scaling studies of high-power fiber lasers, the role of thermally induced effects acquires very much attention, because standard fiber designs would eventually lead to high core temperature $[9,10]$ and the usual assumption of neglecting the thermal effects can no longer be justified.

Eccentric-core fiber lasers are important because they have more efficient pump absorption with respect to conventional fiber lasers [11]. The temperature distribution in off-set solid-state laser, whose pump region has been slightly displaced, with respect to crystal axis, has been studied in the literature [12]. Here we consider eccentric-multi-core fiber lasers whose cores are off-set with respect to the cladding center. We consider heat deposited in the core regions which are off with respect to the cladding center. The inhomogeneous heat equation has been solved by the Green function method and the heat behavior in the core and cladding region has been investigated.

\section{Derivation of Green function for fiber lasers}

The general form of the steady-state heat conduction equation is as following:

$$
k \nabla^{2} T(r, \varphi, z)=-Q(r, \varphi, z),
$$

where $T(r, \varphi, z)$ is the temperature distribution, $Q(r, \varphi, z)$ is the heat density and $k$ is the heat conduction coefficient. We consider the heat deposited in an

\footnotetext{
* corresponding author; e-mail: agharaati@pnu.ac.ir
}

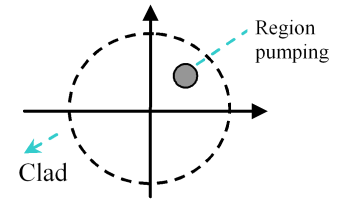

Fig. 1. The cross-section of the fiber laser with the pump in the off-axis position.

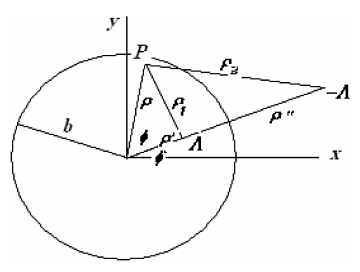

Fig. 2. The cross-section of the fiber laser with pump source $\Lambda$ and its image $-\Lambda$.

out-of-axis region, Fig. 1. We used the Green function method to solve Eq. (1). The Green function can be calculated with analogy to the Poisson equation in electromagnetism. Therefore, by considering a linear heat source, $\Lambda$ inside the fiber, located at $\left(\rho^{\prime}, \varphi^{\prime}\right)$, and its image, $-\Lambda$ at $\left(\rho^{\prime \prime}, \varphi^{\prime \prime}=\varphi^{\prime}\right)$ (Fig. 2), and by using the method of image for a cylinder in cylindrical coordinate, the Green function can be written as [13, 14]:

$$
\begin{aligned}
& G\left(\rho, \phi, \rho^{\prime}, \varphi^{\prime}\right) \\
& \quad=\ln \left(\frac{\rho^{2} \rho^{\prime 2}+b^{4}-2 \rho \rho^{\prime} b^{2} \cos \left(\varphi-\varphi^{\prime}\right)}{b^{2}\left[\rho^{2}+\rho^{\prime 2}-2 \rho \rho^{\prime} \cos \left(\varphi-\varphi^{\prime}\right)\right]}\right),
\end{aligned}
$$

where $(\rho, \varphi)$ is an arbitrary observation coordinate inside the fiber.

We consider the Dirichlet boundary condition in which the temperature is identified on the boundary. Therefore, 
the temperature distribution can be written as

$$
\begin{aligned}
& T(\rho, \varphi)=\frac{1}{4 \pi k} \iint Q\left(\rho^{\prime}, \varphi^{\prime}\right) G\left(\rho, \varphi, \rho^{\prime}, \varphi^{\prime}\right) \rho^{\prime} \mathrm{d} \rho^{\prime} \mathrm{d} \varphi^{\prime} \\
& -\frac{1}{4 \pi} \oint T\left(b, \varphi^{\prime}\right) \frac{\partial G\left(b, \varphi, \rho^{\prime}, \varphi^{\prime}\right)}{\partial \rho^{\prime}} b \mathrm{~d} \varphi^{\prime} .
\end{aligned}
$$

where the second integral is evaluated on the boundary.

For multi-core fiber, the heat generation in the core is expressed as

$$
\begin{aligned}
& Q\left(\rho^{\prime}, \varphi^{\prime}\right)=Q_{0 i} \text { for } a_{i}-c_{i} \leq \rho^{\prime} \leq a_{i}+c_{i}, \\
& \quad \text { and } \varphi_{i}^{\prime}-\cos ^{-1}\left(s_{i}\right) \leq \varphi^{\prime} \leq \varphi_{i}^{\prime}+\cos ^{-1}\left(s_{i}\right) ; \\
& \text { where } i=1, \ldots, N ; \\
& Q\left(\rho^{\prime}, \varphi^{\prime}\right)=0 \text { otherwhere, }
\end{aligned}
$$

where

$$
s_{i}=\frac{\rho^{2}+a_{i}^{2}-c_{i}^{2}}{2 \rho^{\prime} a_{i}} .
$$

Here $c_{i}$ and $a_{i}$ are $i$-th core radius and radial position, respectively. In the following, we consider a few examples.

\subsection{Single-end pump}

By considering $N=1$ in (4) and by setting the core on the $x$ axis at distance $a$, Fig. 3 , the heat power density

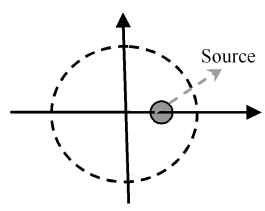

Fig. 3. A single-core fiber laser cross-section.

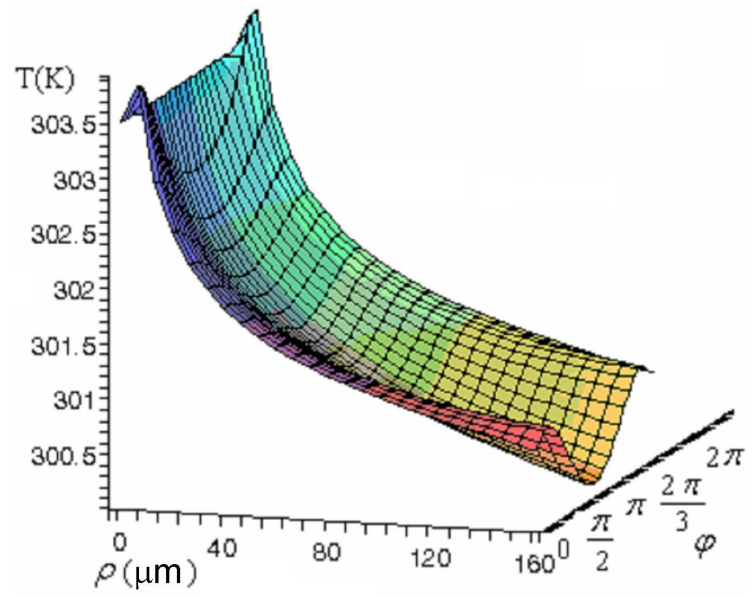

Fig. 4. Temperature distribution function for a single-core fiber laser.

can be written as

$$
\begin{aligned}
& Q\left(\rho^{\prime}, \varphi^{\prime}\right)=Q_{0} \quad \text { for } a-c \leq \rho^{\prime} \leq a+c \\
& \text { and }-\cos ^{-1}(s) \leq \varphi^{\prime} \leq \cos ^{-1}(s) \\
& Q\left(\rho^{\prime}, \varphi^{\prime}\right)=0 \quad \text { otherwhere. }
\end{aligned}
$$

By inserting (6) into (3), and by using the following data: $b=300 \mu \mathrm{m}, c=5 \mu \mathrm{m}, a=20 \mu \mathrm{m}, k=0.051 \mathrm{~W} / \mathrm{cm}^{2} \mathrm{~K}$ and $P_{0}=200 \mathrm{~W}$, the temperature distribution can be plotted as in Fig. 4.

In this case we assume that the surface temperature of the fiber will be $T(b, \phi)=T_{2} \cos (\phi)+T_{1}$.

\subsection{Double core, symmetric case}

In this case, we consider a double-core fiber laser with cores symmetrically located, as depicted in Fig. 5.

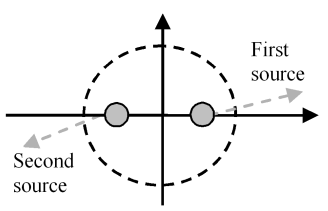

Fig. 5. Symmetric double-core fiber laser cross-section.

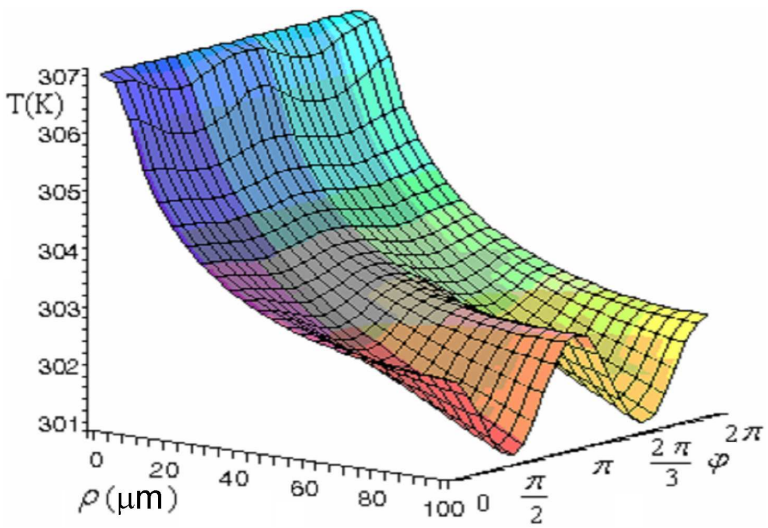

Fig. 6. Temperature distribution function for a symmetric double-core fiber laser.

Therefore, (4) for $N=2$ and $c_{1}=c_{2}$ and $a_{1}=a_{2}$ will be

$$
\begin{aligned}
& Q\left(\rho^{\prime}, \varphi^{\prime}\right)=Q_{0} \quad \text { for } a-c \leq \rho^{\prime} \leq a+c \\
& \quad-\cos ^{-1}(s) \leq \varphi^{\prime} \leq \cos ^{-1}(s) \\
& \text { and } \pi-\cos ^{-1}(s) \leq \varphi^{\prime} \leq \pi+\cos ^{-1}(s) \\
& Q\left(\rho^{\prime}, \varphi^{\prime}\right)=0 \text { otherwhere. }
\end{aligned}
$$

By using previous data, the temperature distribution is plotted in Fig. 6.

In order to calculate the temperature distribution in this case, the following boundary condition has been used: $T(b, \phi)=a+b \cos (2 \varphi)$, where $a$ and $b$ are constants.

\subsection{Double core, asymmetric case}

In this case, the sources are located at $4 \pi / 15$ and $7 \pi / 6$ with respect to $x$-direction, as shown in Fig. 7 . 


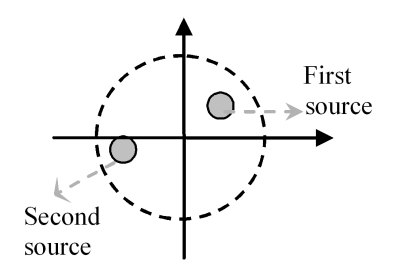

Fig. 7. Asymmetric double-core fiber laser cross-section.

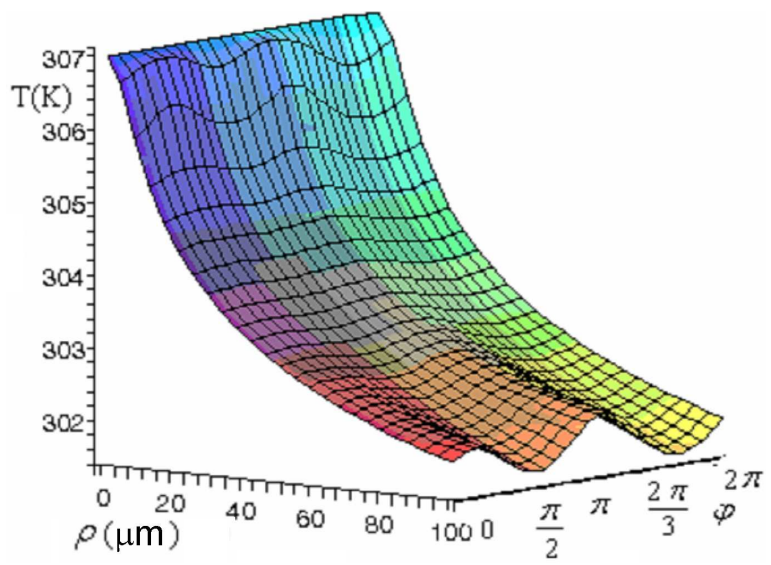

Fig. 8. Temperature distribution function for an asymmetric double-core fiber laser.

So we have

$$
\begin{aligned}
& Q\left(\rho^{\prime}, \varphi^{\prime}\right)=Q_{0} \quad \text { for } a-c \leq \rho^{\prime} \leq a+c ; \\
& 4 \pi / 15-\cos ^{-1}(s) \leq \varphi^{\prime} \leq 4 \pi / 15+\cos ^{-1}(s) \\
& \text { and } 7 \pi / 6-\cos ^{-1}(s) \leq \varphi^{\prime} \leq 7 \pi / 6+\cos ^{-1}(s), \\
& Q\left(\rho^{\prime}, \varphi^{\prime}\right)=0 \text { otherwhere. }
\end{aligned}
$$

The temperature distribution will be as shown in Fig. 8.

In the case of two asymmetrically positioned sources we assume the temperature on the boundary to be (empirically) $T(b, \varphi)=T_{1 b}+T_{2 b} \sin (2 \varphi)+T_{3 b} \cos (2 \varphi)$, where $T_{1 b}, T_{2 b}$ and $T_{3 b}$ are constants.

\subsection{Four cores, asymmetric case}

The cross-section of the fiber laser with four asymmetrically positioned cores is presented in Fig. 9.

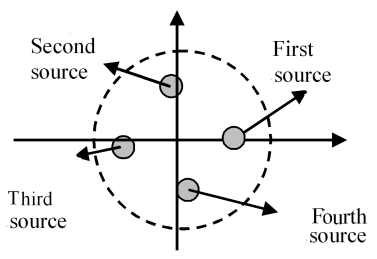

Fig. 9. The cross-section of the fiber laser with four cores asymetrically positioned.
The cores are located at angles $0,53 \pi / 100,103 \pi / 100$ and $8 \pi / 5$ and the temperature distribution function for this case is drawn in Fig. 10.

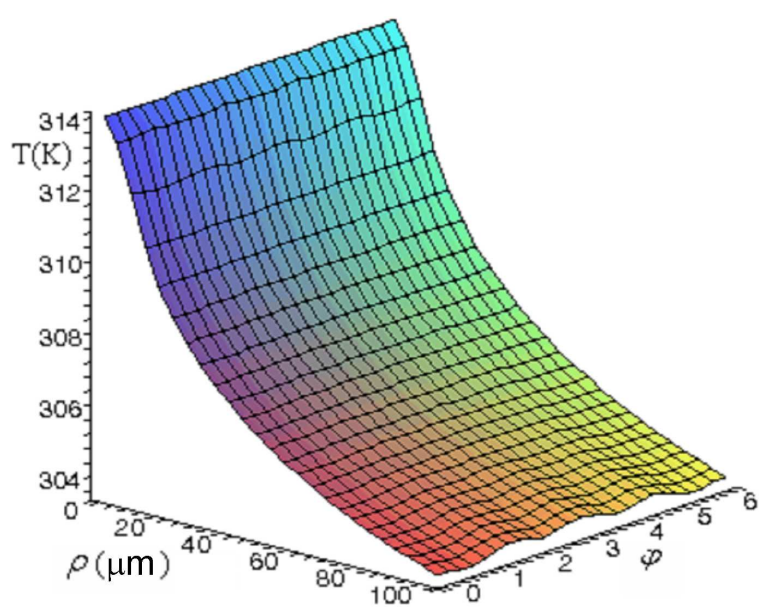

Fig. 10. Temperature distribution in fiber laser with four asymmetrically positioned cores.
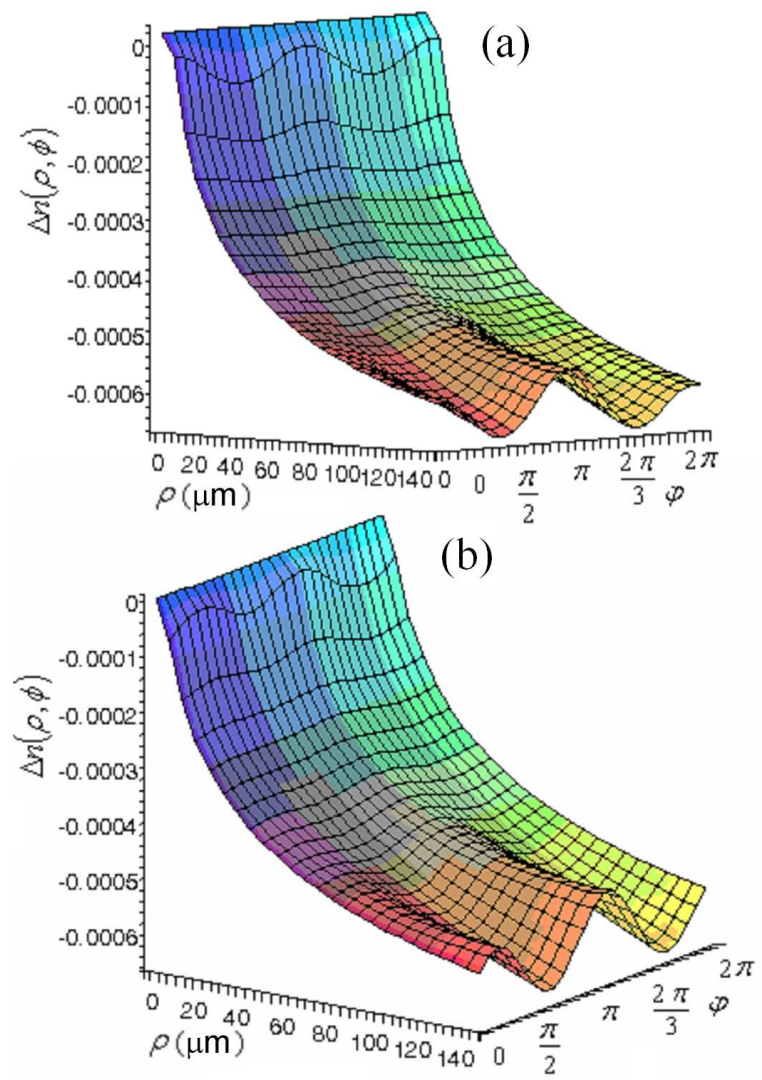

Fig. 11. The variation of refractive index in symmetric and asymmetric double-core fiber lasers.

For this kind of model, the temperature distribution function on the boundary is empirically taken as $T(b, \varphi)=m+n \cos (4 \varphi)$ where $m$ and $n$ are constants. 


\section{Change of refractive index}

In this section, as the application of previous calculations, we are going to find the variation of the refractive index for two double-core fibers, with symmetrically and asymmetrically positioned sources. The variation of the refractive index can be found from $[12,15]$ :

$$
\Delta n(\rho, \phi)=[T(\rho, \phi)-T(0, \phi)] \frac{\mathrm{d} n}{\mathrm{~d} T},
$$

where $\Delta n(\rho, \phi)$ is the differential of the refractive index with respect to the fiber axis and $\mathrm{d} n / \mathrm{d} T$ depends on the fiber material and taken as $\mathrm{d} n / \mathrm{d} T=10^{-5} \mathrm{~K}^{-1}$. Therefore, by using the temperature distribution in symmetric and asymmetric double-core cases, the refractive index change is calculated and plotted in Fig. 11.

\section{Conclusion}

In this paper, the temperature distribution in multi-core fiber lasers has been studied. Because the fiber lasers do not have the axial symmetry, the temperature becomes dependent on the radius and angle coordinates of the cross-section. It can be observed in all of the figures that the temperature is maximal in the core axis, and decreases to the clad temperature at the core boundary. Also, it can be observed that the temperature decreases to the environment temperature at the clad boundary.

Therefore, by finding the temperature dependence in the medium, all optical properties of the fiber can be studied. In this paper, the refractive index and its dependence on the temperature has been studied. Maximal variations of the refractive index have been noticed in the pump region.

\section{References}

[1] D.L. DiGiovanni, M.H. Muendel, Opt. Photonics News, 26 (Jan. 1999).

[2] B. Rossi, Laser Focus World 33, 143 (1997).

[3] D. Richardson, H. Offerhaus, J. Nilson, A. Grudinin, Laser Focus World 35, 92 (1999).

[4] B. Desthieux, R.I. Laming, D.N. Payne, App. Phys. Lett 63, 586 (1993).

[5] R.M. Percival, D. Szebesta, J.R. Williams, R.D.T. Lauder, A.C.!Tropper, D.C. Hanna, Electron Lett. 30, 1598 (1994).

[6] H. Takara, A. Takada, M. Saruwatari, IEEE Photonics. Tech. $\tilde{L}$ 4, 241 (1992).

[7] G. Nykolak, S.A. Kramer, J.R. Simpson, D.J. DiGiovanni, C.R. Giles, H.M. Presby, IEEE Photonics Tech. 3, 1079 (1991).

[8] W.H. Cheng, J.H. Bechtel, Electron. Lett. 29, 2055 (1993).

[9] D.C. Brown, H.J. Hoffman, IEEE J. Quantum Electron. 37, 207 (2001).

[10] H. Nadgaran, P. Elahi, Pramana J. Phys. 65, 95 (2005).

[11] A. Clarkson, in: ICTP Winter College on Fiber Optics, Fiber Lasers and Sensors, Trieste $200 \%$.

[12] P. Elahi, A. Taghavi, A. Gharaati, Pramana J. Phys. 70, 649 (2008).

[13] D. Jackson, Classical Electrodynamics, 3rd ed., Wiley, New York 1998.

[14] D.G. Duffy, Green's Functions with Applications, Chapman \& Hall/CRC, Boca Raton 2001.

[15] W. Koechner, Solid State Laser Engineering, 5th ed., Springer-Verlag, New York 1999. 